\title{
Distribution of Miss Distance in Discrete-Time Controlled System with Noise-Corrupted State Information
}

\author{
Shengwen Xiang, Hongqi Fan, Qiang Fu \\ ATR Laboratory, National University of Defense Technology, Changsha, China \\ Email: xiangsw224@163.com
}

How to cite this paper: Xiang, S.W., Fan, H.Q. and Fu, Q. (2017) Distribution of Miss Distance in Discrete-Time Controlled System with Noise-Corrupted State Information. World Journal of Engineering and Technology, 5, 69-77.

https://doi.org/10.4236/wjet.2017.54B008

Received: September 3, 2017

Accepted: October 9, 2017

Published: October 12, 2017

\begin{abstract}
Miss distance is an important parameter of assessing highly maneuvering targets interception. Due to the noise-corrupted measurement and the fact that not all the state variables can be directly measured, the miss distance becomes a random variable with a priori unknown distribution. Currently, such a distribution is mainly evaluated by the method of Monte Carlo simulation. In this paper, an analytic approach is obtained in discrete-time controlled system with noise-corrupted state information. The system is subject to a bang-bang control strategy. The analytic distribution is validated through the comparison with Monte Carlo simulation.
\end{abstract}

\section{Keywords}

Pursuit-Evasion Game, Highly Maneuvering Target, Miss Distance, Analytic Approach

\section{Introduction}

With the emergence of maneuverable tactical ballistic missiles (TBM) since the 1991 Gulf War, great challenge is put on the air-defense system. Successful interception of such a highly maneuvering target requires a very small miss distance or even direct hit [1] [2]. Due to the bounded missile controls, non-Gaussian noise and system's nonlinearity, the certainty equivalence theorem (CEP) does not hold for the terminal guidance [3]. However, when the relative state between pursuer and evader is observable, the partial separation theorem still holds, when the estimator can design separately from guidance law [4].

The homing guidance of an interceptor missile is a stochastic optimal control 
problem with the objective to minimize the expected value of the miss distance. Under the assumption that perfect state information is available, a series of deterministic control strategies $u=u(t, z(t)) \quad(z(t)$ means ZEM, zero-effort miss distance and its terminal value is actual miss distance itself) are proposed, among them are bang-bang strategies, as well as various linear, saturated linear strategies [5] [6] [7]. However, in practical application perfect state information could not hold, on the one hand, there is a certain error in the observed information of the sensor, and even if the above measurement is very accurate, the target acceleration (especially lateral acceleration) cannot be directly measured and only relays on the means of state estimation; On the other hand, the guidance law like OGL (Optimal Guidance Law) unconstrained the range of missile acceleration, which is bounded in reality. These facts impede significantly the implementation of theoretically robust transferring strategies, in order to use these noise-corrupted measurements as a basis of a feedback control, an estimator, restoring and filtering the state variables, becomes an indispensable component of control loop. Therefore, the control function $u(t, z(t))$ receives, instead of the accurate value of $z(t)$, a random output $\hat{z}(t)=z(t)+\eta(t)$, where $\eta(t)$ is the estimation error of ZEM. As a consequence, the miss distance becomes a prior unknown variable.

To guarantee guidance performance and evaluate the extent of performance deterioration by using such a stochastic data, it is necessary to obtain the distribution of miss distance. In current practice, such a distribution is acquired by a large set of Monte Carlo simulations with system dynamic, estimator/control strategy combination, specific disturbance and noise model given. Unfortunately, this method is very suitable for validate purpose while not proper in the stage of system design. With the assumption that the distributions of $\eta(t)$ and initial state $z_{0}$ are given, Glizer and Shinar [8] have obtained the recursive formula for the distribution of miss distance in discrete-time controlled system with disturbance and noise corrupted measurements under a linear saturated control strategy. In this paper, a recurrence formula for the distribution of $z_{n+1}$ is obtained under a bang-bang control strategy. The use of bang-bang control strategy is motivated by its feature that the maximum capture zone of missile would reach. At the same time, the analytic distribution of $\eta(t)$ in this case is also acquired.

The rest of the paper is organized as follows: Section 2 formulates the estimation problem of terminal guidance for highly maneuvering target interception. Section 3 is devoted to the derivation of MD's distribution. Section 4 validates the above derivation by comparing the results with Monte Carlo simulations. Conclusions are presented in the last section.

\section{Problem Formulation}

\subsection{Continuous-Time Motion Model}

Consider the planar interception scenario-a pursuer (interceptor) and an evader (target), as shown in Figure 1. In what follows, let $\mathrm{P}$ and $\mathrm{E}$ denote the pursuer 


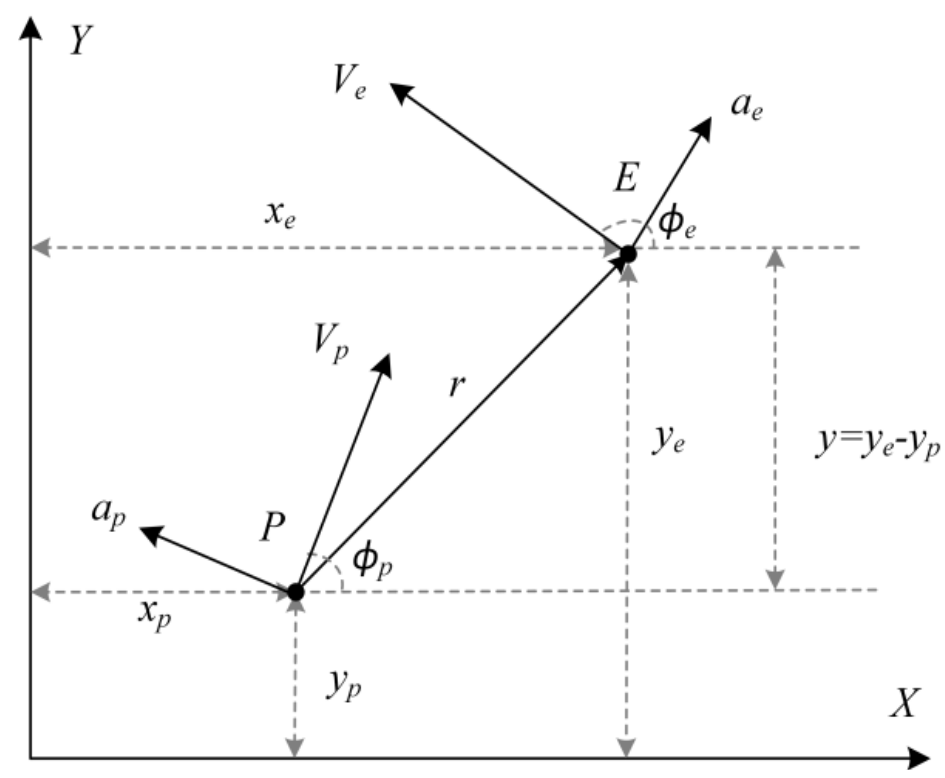

Figure 1. Planer interception geometry.

and evader respectively, and we make the following assumptions:

1) The control dynamics of $P$ and $E$ are expressed by a first-order transfer function with time constants $\tau_{p}$ and $\tau_{e}$ respectively;

2) The velocities of $\mathrm{P}$ and $\mathrm{E}$ are constant, denoted by $V_{p}$ and $V_{e}$ respectively;

3) The lateral accelerations of $P$ and $E$ are both bounded, with maximum values $a_{p}^{\max }$ and $a_{e}^{\max }$ respectively.

In Figure 1, the $X$ axis of coordinate system is aligned with the initial line of sight (LOS); the $Y$ axis is normal to $X$ axis; $\left(x_{p}, y_{p}\right),\left(x_{e}, y_{e}\right)$ are current coordinates of the $\mathrm{P}$ and $\mathrm{E} ; \phi_{p}$ and $\phi_{e}$ are respective aspect angles between the velocity vector of the players and $X$ axis. Note that these aspect angles satisfy such conditions $\left(\sin \phi_{p}=\phi_{p}, \sin \phi_{e}=\pi-\phi_{e}\right)$, the trajectories can linearize along the initial LOS. Assuming a constant closing velocity, let the initial time $t_{0}=0 \mathrm{~s}$ then the final time of the interception can be easily computed for a given initial relative distance $r_{0}$ along with LOS, i.e. the final time is as follows

$$
t_{f} \approx \frac{r_{0}}{V_{p} \cos \phi_{p}(0)-V_{e} \cos \phi_{e}(0)}
$$

Let denote the state vector $\mathbf{x}=\left[x_{1}(t), x_{2}(t), x_{3}(t), x_{4}(t)\right]^{T}=\left[y(t), \dot{y}(t), a_{y}^{e}(t), a_{y}^{p}(t)\right]^{T}$, and for brevity, the time $t$ is omitted hereafter. Under above assumptions, the interception takes place between $t \in\left[0, t_{f}\right]$ and the dynamics of interception has a linear form:

$$
\begin{array}{ll}
\dot{x}_{1}=x_{2}, & x_{1}(0)=0, \\
\dot{x}_{2}=x_{3}-x_{4}, & x_{2}(0)=V_{e} \phi_{e}(0)-V_{p} \phi_{p}(0), \\
\dot{x}_{3}=\left(a_{e}^{c}-x_{3}\right) / \tau_{e}, & x_{3}(0)=0 \\
\dot{x}_{4}=\left(a_{p}^{c}-x_{4}\right) / \tau_{p}, & x_{4}(0)=0
\end{array}
$$


where $\dot{x}_{1}=y_{e}-y_{p}$ is the relative distance between $\mathrm{P}$ and $\mathrm{E}$ normal to LOS; $x_{2}$ is the relative lateral velocity; $x_{3}$ and $x_{4}$ are the lateral accelerations of $\mathrm{P}$ and $\mathrm{E} ; a_{p}^{c}$ and $a_{e}^{c}$ are the commanded accelerations of the $\mathrm{P}$ and $\mathrm{E}$ respectively, and satisfy

$$
\begin{array}{ll}
a_{p}^{c}=a_{p}^{\max } u, & |u| \leq 1 \\
a_{e}^{c}=a_{e}^{\max } v, & |v| \leq 1
\end{array}
$$

The dynamic model can be rewritten as a state vector form

$$
\dot{\mathbf{x}}=A \mathbf{x}+\mathbf{B}_{1} u+\mathbf{B}_{2} v, \quad \mathbf{x}(0)=\left(0, x_{2}(0), 0,0\right)^{T}
$$

where

$$
A=\left[\begin{array}{cccc}
0 & 1 & 0 & 0 \\
0 & 0 & 1 & -1 \\
0 & 0 & \frac{-1}{\tau_{e}} & 0 \\
0 & 0 & 0 & \frac{-1}{\tau_{p}}
\end{array}\right], \quad \mathbf{B}_{1}=\left[\begin{array}{c}
0 \\
0 \\
0 \\
\frac{a_{p}^{\max }}{\tau_{p}}
\end{array}\right], \quad \mathbf{B}_{2}=\left[\begin{array}{c}
0 \\
0 \\
\frac{a_{e}^{\max }}{\tau_{e}} \\
0
\end{array}\right]
$$

By using the scalarizing transformation, the planar interception problem will reduce to a scalar one. In this case, the system variable in the interceptor is $z(t)$, zero-effort miss distance, which means the miss distance acquired with no control effort until the final time $t_{f}$ reached. It can be formulated as

$$
z(t)=\mathbf{D}^{T} \Phi\left(t_{f}, t\right) \mathbf{x}(t)
$$

where $\mathbf{D}=[1,0,0,0]^{\mathrm{T}}, \Phi\left(t_{f}, t\right)$ is the state transition matrix and subjects to the original homogeneous system $\dot{\mathbf{x}}=A \mathbf{x}$,

$$
\Phi\left(t_{f}, t\right)=e^{A\left(t_{f}-t\right)}
$$

Therefore, the zero-effort miss distance is

$$
z(t)=\mathbf{g}^{T}(t) \mathbf{x}(t)
$$

where $\mathbf{g}(t)=\left[g_{1}(t), g_{2}(t), g_{3}(t), g_{4}(t)\right]^{\mathrm{T}}$, with

$$
\begin{aligned}
& g_{1}(t)=1 \\
& g_{2}(t)=t_{f}-t \\
& g_{3}(t)=\tau_{e}^{2}\left\{e^{-\left(t_{f}-t\right) / \tau_{e}}+\left(t_{f}-t\right) / \tau_{e}-1\right\} \\
& g_{4}(t)=-\tau_{p}^{2}\left\{e^{-\left(t_{f}-t\right) / \tau_{p}}+\left(t_{f}-t\right) / \tau_{p}-1\right\}
\end{aligned}
$$

The zero-effort miss distance varies in accordance with the following differential equation:

$$
\dot{z}=h_{1}(t) u+h_{2}(t) v, \quad z\left(t_{0}\right)=z_{0}
$$

where

$$
\begin{gathered}
h_{1}(t)=-a_{p}^{\max } \tau_{p}\left\{e^{-\left(t_{f}-t\right) / \tau_{p}}+\left(t_{f}-t\right) / \tau_{p}-1\right\} \\
h_{2}(t)=a_{e}^{\max } \tau_{e}\left\{e^{-\left(t_{f}-t\right) / \tau_{e}}+\left(t_{f}-t\right) / \tau_{e}-1\right\}
\end{gathered}
$$


It is assumed that the control is given by a bang-bang strategy

$$
u(t, z)=\operatorname{sign}\{z(t)\}
$$

where

$$
\operatorname{sign}(x)= \begin{cases}1, & x>0 \\ -1 & x<0\end{cases}
$$

\subsection{Discrete-Time Estimation Problem}

Define the division of interval $\left[0, t_{f}\right]: 0=t_{0}<t_{1}<\ldots<t_{N}=t_{f}$, where $t_{n+1}-t_{n}=\Delta t$, $n=0,1, \ldots, N-1$. For the sake of simplicity, it is assumed that the system is disturbance free, that is $v=0$, then the equivalent discrete-time Equation of (8) is

$$
z_{n+1}=z_{n}+b_{n} u_{n}
$$

where $b_{n}=h_{1}\left(t_{n}\right) \cdot \Delta t$ and the control

$$
u_{n}=\operatorname{sign}\left(z_{n}+\eta_{n}\right)
$$

In our previous work, we have acquired the recursive expression of the ZEM estimation error distribution. It can be reached that $\eta_{n}$ is subject to Gaussian distribution at every time with mean $\mu_{n}$ and variance $\sigma_{n}^{2}$, i.e., $\eta_{n} \sim N\left(\mu_{n}, \sigma_{n}^{2}\right)$. In this case, $\mu_{n}$ and $\sigma_{n}^{2}$ satisfy

$$
\begin{gathered}
\mu_{n+1}=\mathbf{g}^{T}{ }_{n+1}\left(I-K_{n+1} H\right) \Phi \boldsymbol{\xi}_{n} \\
\sigma_{n+1}^{2}=\mathbf{g}_{n+1}^{T}\left\{\left(I-K_{n+1} H\right)\left(\Phi \Sigma_{n} \Phi^{\mathrm{T}}+Q_{n}\right)\left(I-K_{n+1} H\right)^{T}+K_{n+1} R_{n+1} K_{n+1}^{\mathrm{T}}\right\} \mathbf{g}_{n+1}
\end{gathered}
$$

where $K_{n}$ is the current Kalman gain; $H$ is the measurement matrix; $Q_{n}$ and $R_{n}$ are the covariance matrix of process noise and measurement noise respectively; $\xi_{n}$ and $\Sigma_{n}$ are the mean and covariance matrix of state estimation error, which satisfy

$$
\begin{gathered}
\xi_{n+1}=E\left\{\hat{\mathbf{x}}_{n+1}-\mathbf{x}_{n+1}\right\}=\left(I-K_{n+1} H\right) \Phi \xi_{n}, \quad \xi_{0}=\tilde{\mathbf{x}}_{0} \\
\Sigma_{n+1}=\operatorname{cov}\left\{\hat{\mathbf{x}}_{n+1}-\mathbf{x}_{n+1}\right\}=\left(I-K_{n+1} H\right)\left(\Phi \Sigma_{n} \Phi^{\mathrm{T}}+Q_{n}\right)\left(I-K_{n+1} H\right)^{\mathrm{T}}+ \\
K_{n+1} R_{n+1} K_{n+1}^{\mathrm{T}}, \quad \Sigma_{0}=\tilde{P}_{0}
\end{gathered}
$$

Therefore, the initial ZEM estimation error satisfies $\eta_{0} \sim N\left(\mu_{0}, \sigma_{0}^{2}\right)$ with $\mu_{0}=\mathbf{g}_{0}^{T} \cdot \tilde{\mathbf{x}}_{0}$ and $\sigma_{0}^{2}=\mathbf{g}_{0}^{T} \cdot \tilde{P}_{0} \cdot \mathbf{g}_{0}$ where $\mathbf{g}_{0}=\mathbf{g}(0)$. Once the initial state of estimator $\hat{\mathbf{x}}(0)$ is given, the distribution of $z_{0}$ can be computed as $z_{0} \sim N\left(\mathbf{g}_{0}^{T} \cdot \hat{\mathbf{x}}_{0}-\mu_{0}, \sigma_{0}^{2}\right)$.

\section{Probability Density Function of $\mathrm{z}_{\mathrm{n}+1}$}

From (15) and (16) it can be seen that $z_{n}$ and $u_{n}$ is dependent, hence the convolution formula cannot be used when calculating the distribution of $z_{n+1}$. The cumulative distribution of $Z_{n+1}$ satisfies

$$
\left.F_{z_{n+1}}(x)=P\left(z_{n+1}<x\right)=p_{1} P\left(z_{n}+\eta_{n}>0\right)+p_{2} P\left(z_{n}+\eta_{n}\right)<0\right)
$$

where

$$
p_{1}=P\left(z_{n}+b_{n}<x \mid z_{n}+\eta_{n}>0\right)
$$




$$
p_{2}=P\left(z_{n}-b_{n}<x \mid z_{n}+\eta_{n}<0\right)
$$

By using the formula for the probability of the product of dependent event, we can obtain

$$
p_{1}=\frac{P\left(z_{n}+b_{n}<x, z_{n}+\eta_{n}>0\right)}{P\left(z_{n}+\eta_{n}>0\right)}
$$

then

$$
p_{1}=\frac{\int_{-\infty}^{x-b_{n}} f_{z_{n}}(y) \int_{-y}^{\infty} f_{\eta_{n}}(s) d s d y}{\int_{0}^{\infty} f_{z_{n}+\eta_{n}}(y) d y}
$$

Note that the random variables $Z_{n}$ and $\mu_{n}$ are independent, therefore

$$
f_{z_{n}+\eta_{n}}(y)=f_{z_{n}}(y) * f_{\eta_{n}}(y)=\int_{-\infty}^{\infty} f_{z_{n}}(y-s) f_{\eta_{n}}(s) d s
$$

where the symbol “ $*$ " means convolution, then

$$
p_{1}=\frac{\int_{-\infty}^{x-b_{n}} f_{z_{n}}(y) \int_{-y}^{\infty} f_{\eta_{n}}(s) d s d y}{\int_{0}^{\infty} \int_{-\infty}^{\infty} f_{z_{n}}(y-s) f_{\eta_{n}}(s) d s d y}
$$

Similarly, the following formula can be obtained

$$
p_{2}=\frac{\int_{-\infty}^{x+b_{n}} f_{z_{n}}(y) \int_{-\infty}^{-y} f_{\eta_{n}}(s) d s d y}{\int_{0}^{\infty} \int_{-\infty}^{\infty} f_{z_{n}}(y-s) f_{\eta_{n}}(s) d s d y}
$$

By substituting (27), (28) into (21) the following formula can be obtained

$$
F_{z_{n+1}}(x)=\int_{-\infty}^{x-b_{n}}\left[f_{z_{n}}(s) \cdot \int_{-s}^{\infty} f_{\eta_{n}}(y) d y\right] d s+\int_{-\infty}^{x+b_{n}}\left[f_{z_{n}}(s) \cdot \int_{-\infty}^{-s} f_{\eta_{n}}(y) d y\right] d s
$$

for $b_{n} \geq 0$, by simplifying (29)

$$
\begin{aligned}
& F_{z_{n+1}}(x)=\int_{-\infty}^{x-b_{n}}\left[f_{z_{n}}(s) \cdot \int_{-s}^{\infty} f_{\eta_{n}}(y) d y\right] d s+\int_{-\infty}^{x-b_{n}}\left[f_{z_{n}}(s) \cdot \int_{-\infty}^{-s} f_{\eta_{n}}(y) d y\right] d s \\
& +\int_{x-b_{n}}^{x+b_{n}}\left[f_{z_{n}}(s) \cdot \int_{-\infty}^{-s} f_{\eta_{n}}(y) d y\right] d s \\
& =\int_{-\infty}^{x-b_{n}}\left[f_{z_{n}}(s) \cdot \int_{-\infty}^{\infty} f_{\eta_{n}}(y) d y\right] d s+\int_{x-b_{n}}^{x+b_{n}}\left[f_{z_{n}}(s) \cdot \int_{-\infty}^{-s} f_{\eta_{n}}(y) d y\right] d s
\end{aligned}
$$

from the characteristic of probability density function $\int_{-\infty}^{\infty} f_{\eta_{n}}(y) d y=1$, then

$$
F_{z_{n+1}}(x)=\int_{-\infty}^{x-b_{n}} f_{z_{n}}(s) d s+\int_{x-b_{n}}^{x+b_{n}}\left[f_{z_{n}}(s) \cdot \int_{-\infty}^{-s} f_{\eta_{n}}(y) d y\right] d s
$$

Similarly, for $b_{n}<0$ 


$$
F_{z_{n+1}}(x)=\int_{-\infty}^{x+b_{n}} f_{z_{n}}(s) d s+\int_{x+b_{n}}^{x-b_{n}}\left[f_{z_{n}}(s) \cdot \int_{-s}^{\infty} f_{\eta_{n}}(y) d y\right] d s
$$

By differentiating (31) and (32) with respect to $x$, a same expression for the probability density function of $z_{n+1}$ can be acquired

$$
f_{z_{n+1}}(x)=f_{z_{n}}\left(x-b_{n}\right)+f_{z_{n}}\left(x+b_{n}\right) \cdot \int_{-\infty}^{-x-b_{n}} f_{\eta_{n}}(y) d y-f_{z_{n}}\left(x-b_{n}\right) \cdot \int_{-\infty}^{-x+b_{n}} f_{\eta_{n}}(y) d y
$$

The probability density function $f_{z_{N}}(x)$ is computed by applying the recurrence Formula (33) $N$ times.

\section{Numerical Simulation}

To validate the correctness of theoretical derivation in Section 3, this experiment compares the results of Monte Carlo simulation with the analytic results through a typical instance of TBM interception, where the simulation parameters are listed in Table 1. The number of Monte Carlo simulation is set to be 1000. In this interception scenario, the evader is assumed to move with maximum velocity $V_{e}$, and its command acceleration is subject to uniform distribution between $-0.3 \mathrm{~g}$ and $0.3 \mathrm{~g}$. The initial state estimation errors of $x_{1}(0), x_{2}(0)$ and $x_{3}(0)$ for the estimator are set to be $10 \mathrm{~m}, 5 \mathrm{~m} / \mathrm{s}$ and $1 \mathrm{~g}$ respectively. Cumulative distribution of the miss distance $\left|z_{N}\right|$ is calculated as

$$
F_{\left|z_{N}\right|}(x)=F_{Z_{N}}(x)-F_{Z_{N}}(-x)
$$

where $F_{Z_{N}}(x)=\int_{-\infty}^{x} f_{z_{N}}(y) d y$. Figure 2 shows the result, it is seen that two curves match very accurately.



Figure 2. Theoretical and simulative distribution functions of $\left|z_{N}\right|$. 
Table 1. Simulation parameters.

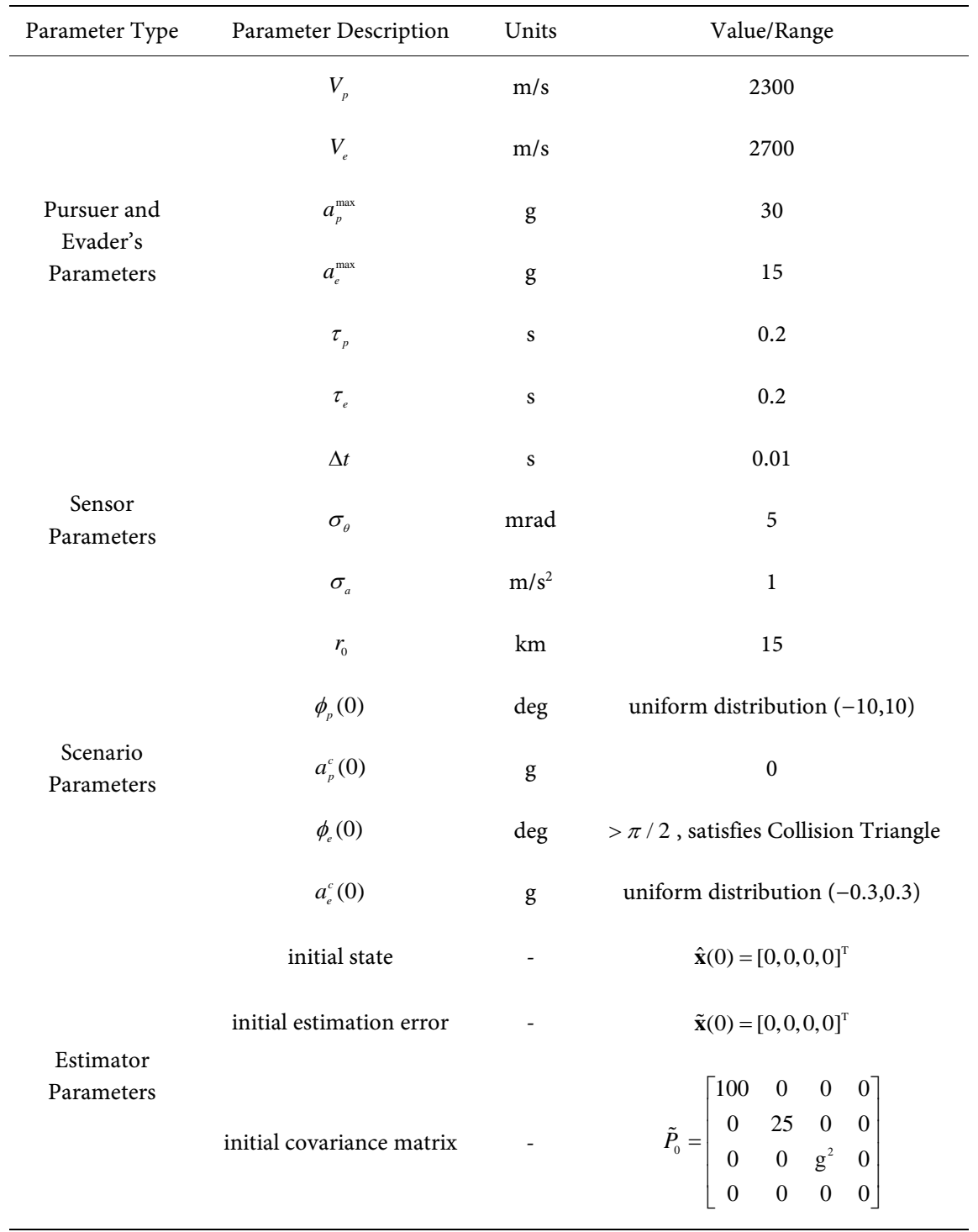

\section{Conclusions}

In this paper, an analytic method for evaluating the probability distribution of miss distance in discrete-time controlled system is proposed. In this problem, the system is assumed without disturbance, and the control is realized by a bang-bang strategy. Results are proved by Monte Carlo simulations in the context of highly maneuvering target interception.

The problem is mathematically nontrivial, because the evaluation of the sum of two dependent random variables is required. Conclusions in this paper allow to evaluate the distribution of miss distance without carrying out a large amount of Monte Carlo simulations.

\section{References}

[1] Shinar, J. and Turetsky, V. (2009) Meeting the Challenges of Modern Interceptor 
Guidance by Non-Conventional Approaches. 17th Mediterranean Conference on Control \& Automation, Makedonia Palace, Greece, 2009, 1563-1568. https://doi.org/10.1109/MED.2009.5164770

[2] Shinar, J., Oshman, Y. and Turetsky, V. (2003) On the Need for Integrated Estimation/Guidance Design for Hit-to-Kill Accuracy. American Control Conference, Inst. of Electronics Engineers, Piscataway, NJ. p., Jun 2003, 402-407.

[3] Witsenhausen, H.S. (1971) Seperation of Estimation and Control for Discrete Time System. Proceedings of IEEE, 59, 1557-1566. https://doi.org/10.1109/MED.2009.5164770

[4] Alexandre, P. (2004) Multiple Model Estimation and Detection for Adaptive Guidance of Hybrid Systems. Master Thesis, McGill University, Montreal.

[5] Shinar, J. (1981) Solution Techniques for Realistic Pursuit-Evasion Games. Advances in Control and Dynamic Systems, 17, 63-124. https://doi.org/10.1016/B978-0-12-012717-7.50009-7

[6] Turetsky, V. and Glizer, V.Y. (2005) Continuous Feedback Control Strategy with Maximal Capture Zone in a Class of Pursuit Games. International Game Theory Review, 7, 1-24. https://doi.org/10.1142/S0219198905000375

[7] Turetsky, V. (2007) Capture Zones of Cheap Control Interception Strategies. Journal of Optimization Theory and Applications, 135, 69-84. https://doi.org/10.1007/s10957-007-9224-y

[8] Glizer, V.Y., Turetsky, V. and Shinar, J. (2012) Terminal Cost Distribution in Discrete-Time Controlled System with Disturbance and Noise-Corrupted State Information. IAENG International Journal of Applied Mathematics, 42, 52-59. 